\title{
Evaluation of Rational Medicine Use Based on WHO Core Drug Use Indicators in Public Hospitals in West Shoa Zone, Oromia, Ethiopia
}

\author{
Esayas Tadesse Gebramariam* and Mustefa Ahmed \\ Unit of Pharmaceutics and Social Pharmacy, Department of Pharmacy, College of Medicine and Health Sciences, Ambo University, \\ Ethiopia
}

\begin{abstract}
Background: The irrational use of medicines is a worldwide problem increasing morbidity, mortality and costs through increasing adverse medicine reactions and hence patients are not achieving their desired outcomes. In Ethiopia, although there are studies that show the presence of irrational use of medicines in the country, there is limited objective evidence on evaluating medicine use from prescriber, patient and health facility perspectives. Therefore, the objective of this study was to evaluate rational medicine use based on WHO-core medicine use indicators in public hospitals in West Shoa zone, Oromia region, Ethiopia.

Methods: Retrospective and prospective cross sectional study were employed to collect data from prescriptions dispensed through main pharmacies and Information for patients respectively. A structured questionnaire was employed to collect information from prescription papers and respondents after obtaining a verbal consent. Data was entered, cleaned, edited and analyzed using SPSS Version 23.0 statistical software package.

Results: The average number of medicines prescribed per encounter was 1.74 . The $\%$ of encounters in which an antibiotic and injection was prescribed was $48.9 \%$ and $12.6 \%$ respectively. The mean consultation time spent between the prescriber and patient was 5.12 minutes and the mean pharmacy dispensing time was 1.28 minutes. The availability of selected tracer medicines in the health facilities was $79.6 \%$.

Conclusion: The study identified that the pattern of prescribing was appreciable and nearly similar with WHO standard reference. However, the patient care factors needs to be improved.

Keywords: Rational drug use; Indicators; Prescriber; Patient; Health facility

Abbreviations and Acronyms: EDL: Essential Drug List; OPD: Out Patient Department; RMU: Rational Medicine Use; SPSS: Statistical Package for Social Sciences; STG: Standard Treatment Guide Line; WHO: World Health Organization
\end{abstract}

\section{INTRODUCTION}

Medicines are substances or a mixture of substances used in the prevention, diagnosis, cure, mitigation or treatment of disease [1]. They are indispensable products in the course of health care service delivery and when available and managed well have been shown to improve customer satisfaction and patient attendance [2]. However, medicine use is a complex activity involving the interaction of different bodies like health professionals, the patient (client) and health institutions. It is influenced by factors such as accessibility of medicines, prescribers' knowledge and experience of health professionals, health budget, and promotional activities of the medicine manufacturing companies [3].

Rational medicine use (RMU) is well recognized as an important part of health policy. It requires that taking appropriate medication by patients to the right clinical needs, in right doses for an adequate period of time, at an affordable price. RMU is achieved when the prescribers used an essential medicines list (EML) to prescribe medicines. According to the World Health Organization (WHO), a

Correspondence to: Esayas Tadesse Gebramariam, Unit of Pharmaceutics and Social Pharmacy, Department of Pharmacy, College of Medicine and Health Sciences, Ambo University, Ethiopia, P. O. Box: 19, Ambo, Ethiopia, Tel-251911925214; E-mail- esayastadesse23@ yahoo.com;

Received: February 15, 2019; Accepted: April 05, 2019; Published: April 15, 2019

Citation: Gebramariam ET, Ahmed M (2019) Evaluation of Rational Medicine Use Based on WHO Core Drug Use Indicators in Public Hospitals in West Shoa Zone, Oromia, Ethiopia. Adv Pharmacoepidemiol Drug Saf 8:1.

Copyright: (C) 2019 Gebramariam ET, et al. This is an open-access article distributed under the terms of the Creative Commons Attribution License, which permits unrestricted use, distribution, and reproduction in any medium, provided the original author and source are credited. 
rational prescription must meet certain criteria such as appropriate indication, appropriate patient, appropriate medicine, appropriate information and appropriate monitoring. Prescribed medicines are categorized as essential medicines if it satisfies the real demands of majority of the population care services using criteria such as riskbenefit ratio, cost effectiveness and quality [4].

Irrational medicine use is a global problem. Worldwide greater than $50 \%$ of all medicines are prescribed and dispensed incorrectly, while $50 \%$ of patients do not take or wrongly take them. Moreover, about $33 \%$ of the world's populations do not get essential medicines [5]. Misuses of medicines are especially common in low income countries. Common types of irrational medicines use includes: poly pharmacy; inappropriate use of antimicrobials, often in inadequate dosage, for non-bacterial infections; over-use of injections when oral formulations would be more appropriate; failure to prescribe in accordance with standard treatment guidelines and inappropriate self-medication for prescription only medicines [6].

Irrational use of medicines can cause inappropriate patient need, and result stock out of medicines and loss of patient confidence in the health care system that lead to reduced access and patient attendance rates. Shortage of essential medicines and inappropriate doses result in serious morbidity and mortality, particularly for paediatrics infections and chronic diseases, such as hypertension, diabetes, epilepsy and mental disorders. Incorrect use and over-use of medicines result wastage of resources and in significant patient harm in terms of poor patient outcomes and adverse medicine reactions. Furthermore, increased antimicrobial resistance and non-sterile injections to the transmission of hepatitis, HIV/AIDS and other blood-borne diseases can appeared due to inappropriate use of antimicrobials [7].

Improving medicine use would have important financial and public health benefits. Furthermore, the magnitude of inappropriate medicine use at community level is often overlooked and few studies address rational medicine use from prescriber, dispenser and consumer's perspective. More research is needed on socio-cultural factors influencing the rational use of medicines, particularly from prescriber, dispenser and consumer's perspective [8].

The context in Ethiopia is not different from the rest of developing countries. There is irrational use of medicines practice throughout the health care system of the country. For instance, according to national assessment done in 2003, the availability of essential medicines was less than $65 \%$ and $8 \%$ of medicines were found to be expired in the same year [9]. Another, retrospective study conducted in Gondar university hospital showed that the average number of medicines prescribed per encounter was 1.76, whereas, the percentage of encounter with antibiotics prescribed was $52.33 \%$, the percentage of encounters with injections prescribed $47.67 \%$, percentage of medicines prescribed by generic name $74.68 \%$ and those prescribed from EML was $82.44 \%$ which was not in line with WHO recommendation [10].

Over all, although there are studies that indicate the presence of irrational use of medicines in the country, there is few studies conducted on RMU in the county and studied the subject from one perspective only; without including WHO recommendation of 3 indicators that is the practice of prescribers, dispensers and patients about RMU. Therefore, the aim of this study was to evaluate rational medicine use based on WHO-core medicine use indicators in public hospitals in West Shoa zone, Oromia regional state, Ethiopia.
In view of the importance of the hospitals to the overall health of nearby peoples, this study will present pooled data on the pattern of prescription medicine use from general outpatient dispensaries of these hospitals and it address key aspects of what the patients experience at hospitals, and how well they have been prepared to deal with the medicine that have been prescribed and dispensed to limit undesired toxicity and adverse events and maximizes on the benefits that can be derived from optimal use of medications. Furthermore, the study is beneficial for zonal and regional health departments and policy makers to take necessary intervention in promoting RMU.

\section{METHODS}

Hospital based cross sectional study design was employed to assess rational medicine use based on WHO core medicine use indicators. Retrospective cross sectional study design was used to evaluate prescribing indicators while prospective cross-sectional design was used for patient care and facility indicators. All outpatient prescriptions dispensed from January 1 to December 31, 2017 period (prescribing indicators); patient attendants and their prescriptions in the hospitals (OPD) from March 1 to March 31, 2018 (patient care indicators) and selected essential medicine based on the morbidity was included as study population. The sample size of the study were calculated based on WHO criteria, at least 100 sample sizes of prescription encounters and outpatient attendants (encounters) are recommended in individual health facility [11]. Therefore, to get a more reliable result (greater point estimate of the population) 300 prescription encounters and 200 samples of patients were assessed in each hospital. Accordingly 2100 prescriptions and 1400 patients were included in the study. Of these, the prescriptions were selected by using systematic random sampling technique and the patients were selected through convenient sampling technique method. For health facility indicators 14 essential medicines were investigated as per WHO recommendation. Data was collected using retrospective and prospective structured observational check list for prescribing and patient care indicators respectively. A reference of patient prescription papers used to check the patient knowledge of how to take correct dosage. Stop watch was used to determine the contact time of health care providers with patient (consultation and dispensing time). Prospective observational checklist was also applied for facility indicators.

After the data was manually checked for completeness and consistencies, it was entered and analyzed by using SPSS version 23. Descriptive statistics including frequency, mean, percentage and table was used to present the data. During data entry Medical supplies were not counted as drugs and anthelmintic and antiprotozoal were not counted as antibiotics. Vaccines including Tetanus antitoxin were not considered as injections. Some commonly used brand names of known drug combinations such as Coartem $^{\circledR}$, Terracortril and Augmentin ${ }^{\circledR}$ were considered as generic names. Abbreviations of drugs such as TTC for tetracycline and CAF for chloramphenicol were considered as generic names. Class names such as antacid and cough syrup were considered as others (neither generic nor brand names). In counting the number of drugs per prescription, known combination therapies like triple therapy for $H$. pylori was not counted as 3 and diuretic, ACEI and spironolactone for cardiac failure was not counted as 3 in recording the number of drugs per prescription.

Ethical approval was obtained from the research and community service team leader of college of medicine and health sciences of 
Ambo University and from the respective hospitals. Besides, a verbal consent was obtained from all participants before starting the actual data collection. Confidentiality and anonymity of information was maintained throughout the data collection and analysis period by not linking personal identifiers in the data presentations.

\section{RESULTS}

A total of seven hospitals were surveyed. A total of two thousand one hundred (2100), prescriptions were used for the evaluation of the prescribing indicators and a total of one thousand four hundred (1400) patients participated in the study regarding consultation and dispensing times, labelling basics and patient's knowledge on correct dosage.

\section{Prescribing indicators}

The prescribing pattern observed in the seven hospitals indicated that a total of 3654 medicines were prescribed from 2100 prescription encounters. The average number of medicines per prescription paper was 1.74 ranging from 1 to 5 per prescription. From the total of 2100 prescription papers evaluated in the study $334(15.8 \%)$ contained one medicines per a single patient, 648 (30.9\%) contained two, $904(43.1 \%)$ contained three and 214 $(10.2 \%)$ contained four or more medicines. The mean number of medicines prescribed per a single patient was not significantly different from that of the WHO standard value i.e. 1.6-1.8 mean per facility.

The numbers of prescriptions containing one or more injectable medicines were $12.6 \%$ in average, with no significant variations among the seven hospitals. The average percentage of patients receiving one or more antibiotics in the hospitals was found to be $48.9 \%$, the higher $(58.6 \%)$ in Enchine and the lower $(38.3 \%)$ in Ambo general hospital. Among all the medicines prescribed in the hospitals $88.3 \%$ were generic medicines and all of them were present in EML (Table 1).

Table 1: Distribution of medicine prescription indicators in seven public hospitals, West Shoa Zone, Ethiopia, March 2018.

\begin{tabular}{ccc}
\hline Prescribing indicators & $\begin{array}{c}\text { Total no of } \\
\text { prescription } \\
(\mathbf{N}=2100)\end{array}$ & $\begin{array}{c}\text { Percentage } \\
(\%)\end{array}$ \\
\hline Number of medicine per prescription & \\
\hline One & 334 & 15.8 \\
\hline Two & 648 & 30.9 \\
\hline Three & 904 & 43.1 \\
\hline Four & 182 & 8.7 \\
\hline Five & 32 & 1.5 \\
\hline Number of medicine encounter with antibiotics & \\
\hline None & 1073 & 51.1 \\
\hline One & 846 & 40.3 \\
\hline Two & 181 & 8.6 \\
\hline Number of medicine encounter with injection & \\
\hline None & 1836 & 87.4 \\
\hline One & 184 \\
\hline Two & 8.8 \\
\hline $\begin{array}{c}\text { Number of medicine prescribed by } \\
\text { generic name }\end{array}$ & 2032 \\
\hline $\begin{array}{c}\text { Number of medicine prescribed from } \\
\text { essential medicine list }\end{array}$ & 2100 \\
\hline
\end{tabular}

Concerning the prescribing patterns in each hospital, the average number of medicines per prescription was 1.62, 1.67, 1.76, 1.73, 1.8, 1.79 and 1.81 in AURH, AGH, GIH, GEH, GUH, JH and EH respectively.

The percentage of medicines encountered with antibiotics were 122 (40.7\%), 115 (38.3\%), 148 (49.4\%), 139(46.4\%), 166 (55.2\%), 161 (53.7\%) and 176 (58.6\%) in AURH, AGH, GIH, GEH, GUH, $\mathrm{JH}$ and $\mathrm{EH}$ respectively. The percentage of medicines prescribed from Ethiopian EML was 100\% in all studied hospitals (Table 2).

\section{Patient care indicators}

Regarding patient care, the study found that the average consultation time was 5.12 minutes. As it can be seen from Table 3 , Jeldu hospital had the lower mean consultation time 4.20 and the higher was in Ambo general hospital i.e.6.35 minutes. The overall mean pharmacy dispensing time was 1.28 minutes.

Among 332 medicines dispensed in all seven hospitals 243 (73.2\%) medicines were actually dispensed to the patients. Alarmingly, no single medicine was adequately labelled ( $0 \%)$, patient name was not indicated in any of the labels, and whereas all of the labels contain dosage form. However, $56 \%$ medicines were fairly labelled, i.e. they contained at least four of the information, drug name, strength, dose and frequency of dosage. The average adequacy of patient knowledge $(n=1400)$ on correct dosage of medicines dispensed was $69.6 \%$ in all selected hospitals (Table 3).

\section{Health facility indicators}

Evaluation of health facility indicators showed that 3 (42.9\%) of hospitals had a copy of Ethiopian EML, 7 (100\%) had a copy of standard treatment guideline (STG) for hospitals and 5 (71.4\%) of the them had a copy of medicine formulary; however others have not any essential reading materials listed above during the study period. the availability of tracer medicines in the studied hospitals was $79.6 \%$ (Table 4 ).

Moreover, tracer medicines like ferrous salts with folic acids, Vitamin A and tetracycline eye ointments were not available in majority of the hospitals during the study period (Table 5).

\section{DISCUSSION}

The WHO prescription indicators provide a reproducible and objective measure for characterizing prescriptions. The guidelines on rational use of drugs had reference values of (1.6-1.8) medicine per encounter; the average of 1.74 medicines in this study is much comparable and within in the acceptable limits [12]. The findings of this study were much lower than similar studies done in different countries like Nigeria, Bangladesh and others, where as the result is comparable with that obtained in Hawasa referral hospital which is 1.9 as reported by Anteneh [12,13]. The lower number of medicines per encounter might be seen in its positive prospect that as the degree of poly pharmacy decreases, low incidences of side effects and drug-drug interactions and enhanced compliance by the patients could be obtained [14].

The percentage of encounters in which antibiotics were prescribed is $48.9 \%$ which is greater than the WHO standard value 20.0-26.8. As this figure indicates that antibiotic prescription is incorrect and it needs regulation. Incorrect use of antibiotics can potentially lead to medicine resistance and increase the necessity to use more expensive antibiotics to treat life-threatening infections $[13,15]$.

In the present study injection use was found to be $12.6 \%$, which is 
Table 2: Average value of medicine prescription indicators as compared to WHO standard value in seven public hospitals, West Shoa Zone, Ethiopia, March 2018.

\begin{tabular}{|c|c|c|c|c|c|c|c|c|c|}
\hline \multirow{2}{*}{ Indicators } & \multirow{2}{*}{$\begin{array}{l}\text { WHO standard } \\
\text { value }\end{array}$} & \multirow{2}{*}{$\begin{array}{l}\text { Study findings } \\
\text { (average) }\end{array}$} & \multicolumn{7}{|c|}{ Mean core medicine indicator in each hospital } \\
\hline & & & AURH & $\mathrm{AGH}$ & GIH & GEH & GUH & $\mathrm{JH}$ & $\mathrm{EH}$ \\
\hline $\begin{array}{c}\text { Average number } \\
\text { of medicines } \\
\text { prescribed }\end{array}$ & $1.6-1.8$ & 1.74 & 1.62 & 1.67 & 1.76 & 1.73 & 1.8 & 1.79 & 1.81 \\
\hline $\begin{array}{l}\% \text { of encounters } \\
\text { with antibiotics }\end{array}$ & $20.0-26.8$ & 48.9 & 40.7 & 38.3 & 49.4 & 46.4 & 55.2 & 53.7 & 58.6 \\
\hline $\begin{array}{l}\% \text { of encounters } \\
\text { with injections }\end{array}$ & 13.4-24.1 & 12.6 & 11.2 & 13.8 & 10.7 & 15.4 & 14.3 & 12.3 & 10.5 \\
\hline $\begin{array}{l}\% \text { of medicines } \\
\text { prescribed by } \\
\text { generic name }\end{array}$ & 100 & 96.7 & 93.7 & 90.7 & 100 & 97.6 & 97 & 98 & 100 \\
\hline $\begin{array}{c}\% \text { medicines } \\
\text { prescribed from } \\
\text { EDL }\end{array}$ & 100 & 100 & 100 & 100 & 100 & 100 & 100 & 100 & 100 \\
\hline
\end{tabular}

AURH-Ambo University Referral Hospital; AGH-Ambo General Hospital, GIH-Gindberet Hospital; GEH-Gedo Hospital; JH-Jaldu Hospital; EHEnchine Hospital

Table 3: Distribution of patient care indicators in seven Public Hospitals, West Shoa Zone, Ethiopia, March 2018.

\begin{tabular}{ccccccccc}
\hline Indicator & Mean value & AURH & AGH & GIH & GEH & GUH & JH & EH \\
\hline $\begin{array}{c}\text { Average consultation } \\
\text { time (min) }\end{array}$ & 5.12 & 6.13 & 6.35 & 4.6 & 5.25 & 4.9 & 4.2 & 1.41 \\
\hline $\begin{array}{c}\text { Average dispensing } \\
\text { time(min) }\end{array}$ & 1.28 & 1.43 & 1.54 & 1.1 & 1.25 & 61.3 & 7.1 \\
\hline $\begin{array}{c}\text { \% medicines actually } \\
\text { dispensed }\end{array}$ & 73.2 & 85.5 & 91 & 67.5 & 0 & 67.5 & 0 \\
\hline $\begin{array}{c}\% \text { of medicines } \\
\text { adequately labeled }\end{array}$ & 0 & 0 & 0 & 0 & 6 & 0 \\
\hline $\begin{array}{c}\% \text { of adequacy of } \\
\text { knowledge of patient }\end{array}$ & 69.6 & 80.5 & 89 & 65 & 62.5 & 67.5 & 60 \\
\hline
\end{tabular}

Table 4: Health facility indicator results in seven hospitals in West Shoa Zone, Ethiopia, March 2018.

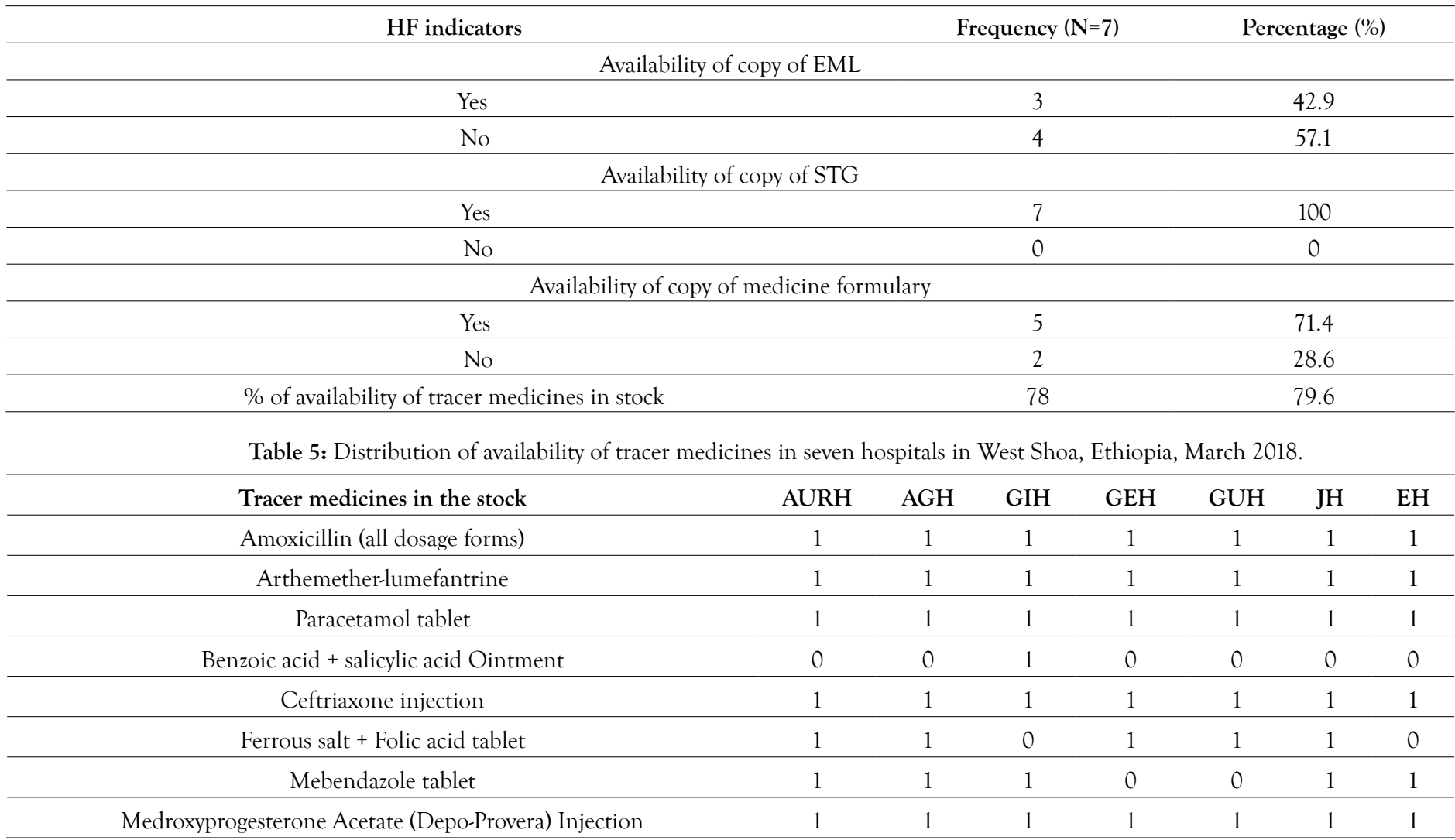




\begin{tabular}{ccccccccc}
\hline Oral rehydration salts & 1 & 1 & 1 & 1 & 1 & 1 & 1 \\
\hline RHZE-table & 1 & 1 & 1 & 1 & 1 & 1 & 1 \\
\hline Tetracycline ointment & 0 & 1 & 0 & 1 & 0 & 0 & 0 \\
\hline Lamivudine + Tenofovir + Efavirenz tablet & 1 & 1 & 1 & 1 & 1 & 1 & 1 \\
\hline Oxytocin injection & 1 & 1 & 1 & 1 & 1 & 0 & 1 \\
\hline Vitamin A (retinol) & 0 & 0 & 0 & 0 & 1 & 0 & 0 \\
\hline Iodine/gentian violet & 1 & 1 & 0 & 0 & 0 & 0 & 0 \\
\hline \% of availability & 85.7 & 92.9 & 78.6 & 78.6 & 78.6 & 71.4 & 71.4 \\
\hline
\end{tabular}

1= Yes, 0=No. AURH-Ambo University Referral Hospital; AGH-Ambo General Hospital, GIH-Gindberet Hospital; GEH-Gedo Hospital; JH-Jaldu Hospital; EH-Enchine Hospital.

closer to the WHO standard value i.e. $13.4 \%-24.1 \%$. This finding is much lower than that obtained from Hawasa referral hospital which was $38.1 \%$, but it is comparable with the report from North West Ethiopia (11.07\%) [10,13]. Low percentage of encounters with injections may have an advantageous in reducing the possibility of transmissions of blood born infections like HIV and hepatitis [16].

WHO guideline expects $100 \%$ of medicines prescription to be generic, similarly in this study $96.7 \%$ of medicines were prescribed by generic names. Prescribing the quality and the cost of prescribed medications can be determined by the level of compliance to generic prescription. Increasing generic prescription could substantially reduce the cost of medicines for patients and health facilities [17].

$100 \%$ of the medicines prescribed in this study were found to be listed in the national EML, even though only three hospitals had a copy of the national EML. A study of the patterns of prescription at Jimma Hospital, south west Ethiopia, showed similar results, where almost all medicines prescribed for the health problems were on the EML of the country, but few medicines prescribed out of the list were found in the national medicine list of Ethiopia $[9,18]$. It is expected that $100 \%$ of medicines will be prescribed from the EML or formulary. Prescribing medicines from the EML issued by WHO provides a framework for rational prescribing; medicines on the list are well-established medicines, already tested in practice, with established clinical use and lower cost than newer medicines [19].

In this study the average consultation and dispensing time in facilities was 5.12 minute and 1.28 minutes respectively. Smaller number of health professionals and large number of patient flow could be the probable reason for such smaller time. The findings from this study were smaller than the study conducted in South West of Ethiopia 6.14 minutes and 1.38 minutes respectively. Moreover, the value was smaller than similar studies conducted in countries like Nigeria and Cambodia [20,21].

According to the recommendation of WHO adequate label of medicines include at least patient name, drug name, strength, dosage (dose and frequency) and duration/quantity of the medicine. In this study none of labels for the medicines contained at least six informations to be said adequately labelled. However, $56 \%$ of drug labels were fairly labelled, fulfilling at least four of the information: drug name, strength, dose and frequency. Even though, high load of patient flow could be one of the factors but, inadequacy of knowledge and skill in labelling of prescribed drugs could be the major factor for these low results. The findings from the study was very much smaller than similar studies conducted locally and other countries, where the smaller figure for adequate labelling was $67 \%$ [18-20].

Regarding percentage of medicines actually dispensed in this study
73.2\% were actually dispensed, this percentage is lower than similar studies conducted in north west Ethiopia and in different other countries like Nigeria and Cambodia. This shows the weakness of the pharmaceutical supply system to provide prescribed medicines to patients. Non-availability of essential medicines or nonadherence to standard treatment protocols could be one reason.

Moreover, the time of data collection was the end of review period in both of the facilities which might lead them in significant stock outs. Whatever the case could be this may also be an indication of poor drug supply management system in the facilities. Because good medicine supply management ensures uninterrupted accessibility of medicines and prevents frequent stock outs and wastages $[17,20$ 22].

In this study, about $70 \%$ of the patients know about the dosage of their medicines at exit interviews. The remaining $30 \%$ of the patients has no information about the use of medicines, which in other words implies that $30 \%$ of the medicines dispensed go waste because of poor communication. Different factors could lead for this barrier such as educational background, cultural, physical factors and etc. Moreover, the short dispensing time by itself could be a factor, because the clients have no enough time to get enough information about the medicines they have been dispensed [23,24].

Majority of the hospitals included in the study were used the national EML, formulary and STGs but none of them had their own EMLs, formularies or STGs. Facility based EML and STG are very crucial for the hospitals since the prevalence of diseases is affected by geographic location of the facilities and socio demographic characteristics of the residents.

About $79.6 \%$ of tracer medicines were in stock. This finding is lower than a result from Nigeria (83.3\%) [19]. The absence of tracer medicines in stock was a strong indicator of weak pharmacy service in the hospitals and impairs the overall services provided by the health sectors [25].

\section{LIMITATIONS OF THE STUDY}

The present study was not described the reasons leading to irrational prescribing of medicines. Moreover, the study was not devised any follow up mechanism to know whether the patient appropriately use the medicines after they have taken the medicines to their home.

\section{CONCLUSION}

The pattern of prescribing was appreciable and nearly similar with WHO standard reference with regard to numbers of medicines prescribed per encounter, percentage of injections prescribed and medicines prescribed from essential medicine list. However, the 
pattern of prescription was far from the WHO standard values regarding to antibiotic prescription. The average consultation and dispensing time was low, which might affect the quality of service given to the patient and there by patient satisfaction. Moreover, above $70 \%$ of prescribed medicines were actually dispensed to the patients and $80 \%$ of tracer medicines were in stock in the hospitals, which are an indicative of low stock outs and good supply management in the hospitals. Almost all guide lines, such as treatment guideline and medicine formularies were available in the hospitals, though none of them had their own EMLs. It is recommended that antibiotic prescription should be in line with the standard treatment guidelines and all medicine dispensed should be adequately labelled.

\section{ACKNOWLEDGEMENT}

We would like to thank Ambo university research and community service office for sponsoring this research project. We are very much thankful to data collectors for their commitment and scrupulous activities they were doing during data collection. We would also like to thank all the participants of the study as this research project would not have been possible without their sincere cooperation.

\section{COMPETING INTERESTS}

The authors of this manuscript declare that they have no competing interests.

\section{REFERENCES}

1. Kishore J. National health programs of India: national policies $\&$ legislations related to health. $6^{\text {th }}$ (edn), Century Publications, New Delhi, India. 2006.

2. Fidler A, Msisha W. Governance in the pharmaceutical sector. Euro health. 2008;14(1):25.

3. WHO. The rational use of drugs: report of a conference of experts. Nairobi, Kenya. 1985.

4. Enwere OO, Falade CO, Salako BL. Drug prescribing pattern at the medical outpatient clinic of a tertiary hospital in southwestern Nigeria. Pharmacoepidemiol Drug Saf. 2007;16(11):1244-1249.

5. Blum NL. Drug information development. A case study Nepal. Rational pharmaceutical management project. United States Pharmacopoeia. 2000.

6. Alam K, Mishra P, Prabhu M, Shankar PR, Palaian S, Bhandari $\mathrm{RB}$, et al. A study on rational drug prescribing and dispensing in outpatients in a tertiary care teaching hospital of Western Nepal. Kathmandu Univ Med J. 2006;4:436-443.

7. Otoom S, Batieha A, Hadidi H, Hasan M, Al- Saudi K. Evaluation of drug use in Jordan using WHO prescribing indicators. East Mediterr Health J. 2002;8(4-5):537-543.

8. FMOH, WHO. Assessment of the pharmaceutical sector in Ethiopia. Ministry of Health, Addis Ababa, Ethiopia. 2003.

9. Admassie E, Begashaw B, Hailu W. Assessment of drug use practices and completeness of prescriptions in Gondar university teaching referral hospital. IJPSR. 2013;4(1):265-275.
10. WHO. How to investigate drug use in health facilities: selected drug use indicators. World Health Organization, Geneva, Switzerland. 1993.

11. Odusanya OO, Oyediran MA. Rational drug use in primary health care centers in Lagos, Nigeria. NigQt J Hosp Med. 2000;10:4-7.

12. Anteneh AD. Assessment of drug use pattern using WHO prescribing indicators at Hawassa University teaching and referral hospital, south Ethiopia: a cross-sectional study. BMC health service research. 2013;13:170.

13. Chima IE. Evaluation of drug use and patient care practices in a referral health facility in Yenagoa, Bayelsa State, Nigeria. Continental J Pharm Sci. 2012;6:10-16.

14. Akinyede AA, Mabadeje AFB, Aliu AA. A comparative study of the patterns of prescription of antibiotics in two health centers in Lagos. J Nig Infect ContAssoc. 2000;3:20-23.

15. Karande S, Sankhe P, Kulkarni M. Patterns of prescription and drug dispensing. Indian J Pediatr. 2005;72(2):117-121.

16. WHO (2010) The world medicines situations: chapter 8, rational use of medicines. World Health Organization, Geneva, Switzerland.

17. Mulugeta TA, Nasir TW, Raju NJ. Assessment of patterns of drug use by using World Health Organization's prescribing, patient care and health facility indicators in selected health facilities in southwest Ethiopia. JAPS. 2011;01(07):62-66.

18. El Mahalli AA. WHO/INRUD drug prescribing indicators at primary health care centres in Eastern province, Saudi Arabia. EMHJ. 2012;18(11):1091-1096.

19. Odusanya OO. Drug use indicators at a secondary health care facility in Lagos, Nigeria. Nig J Comm Med Pri Health Care. 2004;16(1):21-24.

20. Chareonku C, Khun LV, Boonshuyar C. Rational drug use in Cambodia. Study of three pilot health centers in south east in kampong thom province. Southeast Asian J Trop Med Public Health. 2002;33(2):418-424.

21. Management Sciences for Health. Managing Drug Supply. $1^{\text {st }}$ (edn), Kumarian Press, West Hartford, Connecticut, USA. 1997.

22. Lamichhane DC, Giri BR, Pathak OK, Panta OB, Shankar PR. Morbidity profile and prescribing patterns among outpatients in a teaching hospital in western Nepal. Mcgill J Med. 2006;9:126133.

23. Hogerzeil HV, Bimo, Ross-Degnan D, Lang RO, Ofori-Adjei D, Santoso B, et al. Field tests for rational drug use in twelve developing countries. Lancet. 1993;342(8884):1408-1410.

24. Acurcio FA, Perini E, Magalhaes SM, Terceiro LG, Vieira-Filho JM, Coutinho KE, et al. Analysis of medical prescriptions dispensed at health centers in Belo Horizonte, Minas Gerais, Brazil. Cad Saude Publica. 2004;20:72-79.

25. Baghdadi-Sabeti G, Cohen-Kohler JC, Wondemagegnehu E. Measuring transparency in the public pharmaceutical sector: Assessment instrument. World Health Organization. 2009. 